\title{
Experimental and numerical analysis of cylindrical straw drying
}

\author{
Wojciech Goryl ${ }^{1, *}$, Mariusz Filipowicz ${ }^{1}$ \\ ${ }^{1}$ AGH University of Science and Technology, Faculty of Energy and Fuels, A. Mickiewicza Av. 30, 30-059 Krakow, Poland
}

\begin{abstract}
The paper presents experimental and numerical results of the heat and mass transfer in a cylindrical bale of straw. The experimental measurements were made in a specialized stand of straw driers. Flue gasses, comes from straw combustion in the biomass boiler, are used as a drying medium. There were made measurements of humidity and temperature inside the cylindrical straw bale during the drying process. The results were used to prepare the drying rate curve. Moreover, data were used to validate the numerical model of straw drying. The numerical model was performed to depict the heat and mass transfer inside the straw bale. Furthermore, the model was used to optimize the drying process. The paper presents result of experimental and numerical drying rates of cylindrical straw bale and heat and mass transfer in its interior. As a result of the work numerical model was obtained. It satisfactorily describes the mechanisms inside the drying straw bale.
\end{abstract}

\section{Introduction}

Poland, as an agricultural country, and having a large area of forestation has a pretty large biomass resources, which can be used for generating electricity or heat production. One of the basic problems of the energy use of biomass is the bulk density as well as the calorific value. In the given biomass volume there is approx. 4-8 times less energy than in the same coal volume. This causes a number of problems, and the main one is the transportation problem - the costs related to biomass transport over long distances and emissions associated with the biomass transportation from the place of harvesting to the place of utilization. One of the most important factors influencing on the biomass calorific value is humidity. The higher humidity of the biomass the calorific value of biomass is lower. It should be mentioned that there is a lower and upper limit of humidity, which should not be exceeded in energy use of biomass. This has negative effect on biomass combustion process and construction elements of the biomass boiler.

Because of the energy crisis in the 70s, straw has been used on an increasing scale, mainly in Denmark. This country is a precursor of the energy use of straw. The first straw-fired boilers showed very poor working conditions, low efficiency (30-40\%) and high emission of carbon monoxide (above $10000 \mathrm{ppm}$ in the exhaust gas) [1]. Then, in the $80 \mathrm{~s}$ of the $\mathrm{XX}$ century the construction of boilers was improved and the combustion efficiency was achieved within $75 \%$. In the 90s, the Danish government introduced subsidies for the purchase of straw-fired boilers, which resulted in a further development of this fuel. At the beginning of the XXI century the combustion efficiency of $87 \%$ was achieved and the $\mathrm{CO}$ content in the exhaust gases less than $1000 \mathrm{ppm}$ [1].

The easiest way is to use shredded straw in large power boilers, where the fuel moisture is not so relevant $[2,3,4]$. In addition, in the case of shredded straw, drying of the fuel is relatively simple, because the solutions for drying loose materials have been known for many years and they are still used, for example: rotary dryers $[5,6,7]$, pneumatic dryers $[8,9]$, fluidized bed dryers $[10,11,12]$, belt dryers $[12,13,14]$.

The bigger problem is the use of the bales of straw in batch boilers. In this case, drying is not a simple issue [15]. Most studies on drying bales talks about the modification of the structure of the dryer itself, and not the drying process. This study aims to provide an effective and economical process of drying cylindrical bales of straw. Developing an optimised drying process can be performed by conducting long-lasting studies on the experimental stand. However, currently we have got a possibility to apply the advanced computer tools, which assist in conducting numerical calculations. The use of computational fluid dynamics (CFD) [16,17], in this case allows to optimise the drying process without the need to perform multiple series of experimental measurements.

\section{Measurement stand}

The main elements of the dryer used in the study include the drying chamber, the transport table, the discharge fan, the fire prevention system, the regulation assembly and the system for the control of operational parameters, the system of pre-extraction and separation of sparks and the exhaust gasses system.

* Corresponding author: wgoryl@agh.edu.pl 
The heat used in the drying process comes from the process of biomass combustion in the boiler. Such utilised waste heat increases the efficiency of the entire system and helps to obtain a much more efficient fuel. The drying process involves the injection of waste gas characterized by a properly controlled temperature by the automatic system using a specially designed nozzle with a diameter of $80 \mathrm{~cm}$ into the bale of straw.

For safety reasons, the exhaust gases coming from the boiler are pre-dusted in the sedimentation chamber (cyclone) due to the presence of large, sometimes incandescent stalks of unburned straw. Subsequently, the gases are transported to a special mixer (Fig. 1), where, if necessary, the process of mixing of the exhaust gas with ambient air takes place in order to obtain the desired temperature of the drying medium. After the mixing process, the fan introduces the previously prepared mixture using the specially designed nozzle into the cylindrical bale of straw. The schematic diagram of the system has been presented in EFM2015 [18].

The effectiveness of drying cylindrical straw bales (diameter: $160 \mathrm{~cm}$, length: $140 \mathrm{~cm}$ ) with exhaust gases was measured using a specially prepared recorder equipped with eight measuring probes. The probes were calibrated by the manufacturer using a sample of straw characterized by varying humidity. Capacitive relative humidity sensors and temperature sensors with the SHT11 device were installed.

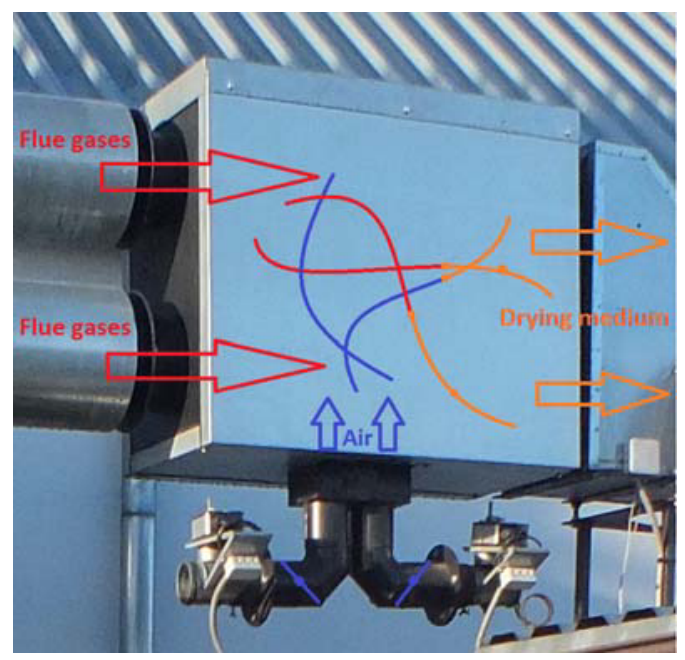

Fig. 1. General view of the medium mixer

The measuring probes varied in length $(80 \mathrm{~cm}, 60$ $\mathrm{cm}, 40 \mathrm{~cm}$ and $20 \mathrm{~cm}$ denoted respectively by lowercase "a", "b", "c" and "d"), so as to make it possible to measure the humidity and temperature in locations at a preset distance from the axis of the straw bale. Figure 2 presents the visualisation of the placement of the right probe in the bale during the measurement.

The measurements were always carried out in two planes. The measurement points were located at a distance $(\mathrm{x}=0$ - the place where the drying mixture was pumped to the bale) of $40 \mathrm{~cm}$ (plane $\mathrm{A}, \mathrm{x}=40$ ) and 100 $\mathrm{cm}$ (plane $\mathrm{B}, \mathrm{x}=100$ ) from one end of the bale (Fig. 2).

The results of the experiment have been presented below. The curves show the change of temperature and humidity during the drying process. The prefixes " $\mathrm{A}$ " and "B" designating the probes refer to the measurement plane according to Figure $2 b$, while the prefixes " $T$ " and " $\mathrm{H}$ " refer to the measurement of temperature and humidity, respectively.

a)

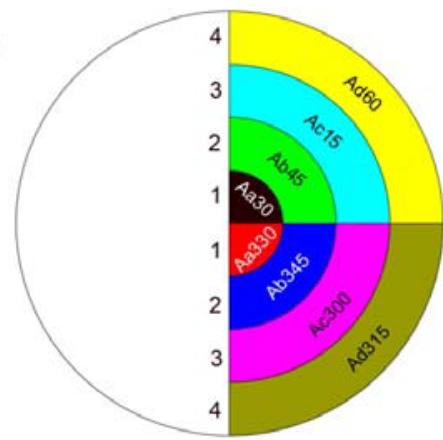

b)

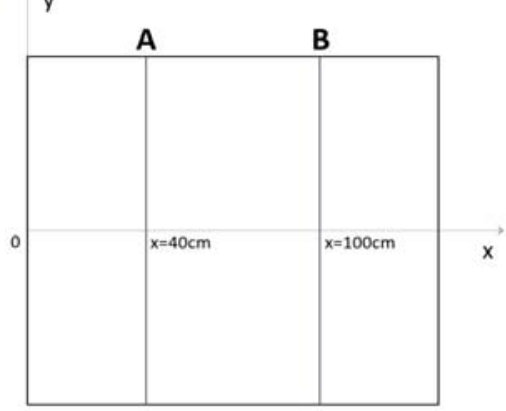

Fig 2. Visualisation of the locations of measurement probes: a) locations of individual probes, b) coordinates of the A and B planes.

This article presents the possibilities of drying straw using a modified drying process. After gaining some experience in the process of drying straw bales $[18,19,20]$, some modifications of this process have been implemented, which involved preparing a duct along the bale axis with the diameter of $63 \mathrm{~mm}$ and applying a metal plate with the diameter of $1,000 \mathrm{~mm}$ at the side opposite to the fitted drying medium. This modification was designed to allow for the highest radial propagation of the medium and to obtain a uniform temperature and humidity inside the bale.

\section{Results and discussion}

A study of changes in the humidity and temperature inside the bale of straw were conducted in the A plane (Fig. 2b), which is presented below. During the drying process, the temperature of the drying medium injected into the bale was recorded. The exhaust gas temperature was presented in Fig. 3-4 to illustrate its dynamics due to operation of the control system. It is presented in the form of a dotted curve with the values given on the right axis. The process have been carried out by $140 \mathrm{~min}$.

The graph below (Fig. 3) presents the change in humidity inside the bale of straw with one hole in the centre of the bale and with the applied metal plate.

The starting humidity of the bale, on which the measurements were performed for the modified process of drying with one hole inside the bale of straw, varied 
between $25-45 \%$. The internal layers of the bale were drier, and the further from the centre of the bale, the humidity started to rise. This was due to the storage of straw uncovered and absorbing moisture by the outer layers.

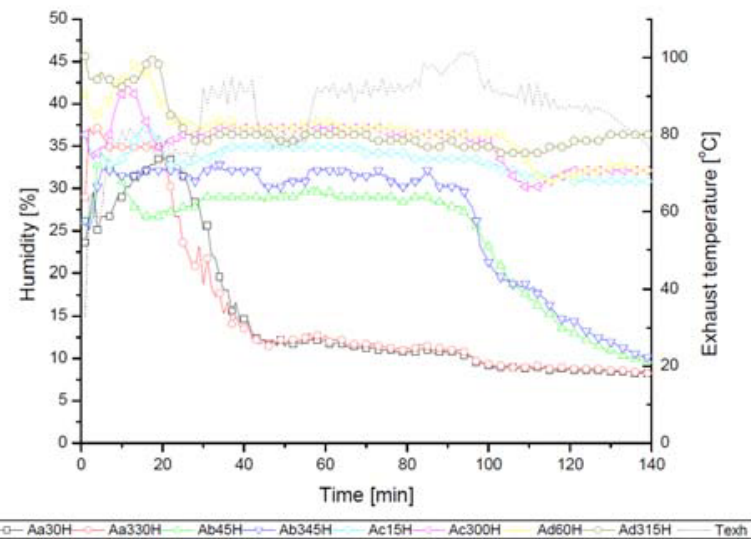

Fig. 3. Humidity measurement.

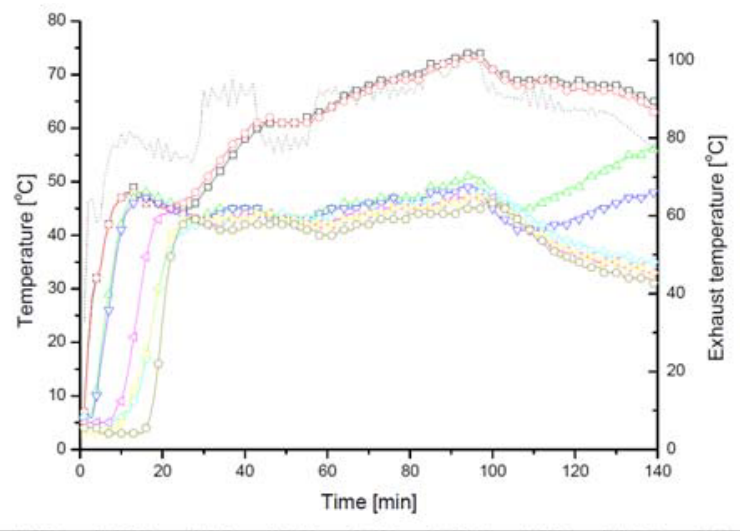

Fig. 4. Temperature measurement.

In the initial phase from approx. $10 \mathrm{~min}$ we can see an increase in moisture in all planes of the bale. This is caused by the rapid rise in temperature in the bale, which contributed to the temporary increase in humidity due to reaching the dew point temperature. After 20 minutes from starting the measurement, the moisture in the entire bale started decreasing. The most rapid drop is visible in Plane 1 and it lasts up to 40 minutes of measurement. Then, moisture in this plane stabilises and at the end of the drying process reaches the value of approx. $8 \%$ of moisture. For Plane 2 to 90 minutes of the process, the moisture remains constant in the range of $28-33 \%$. After 90 minutes, the humidity starts dropping and reaches the values like Plane 1. Plane 3 and 4 behaves similarly like during the drying in the basic drying process, that is, it remains constant in the range of $30-35 \%$.

The above chart (Fig. 4) presents the temperature change inside the bale of straw. After 2 minutes from the start of the drying process we can observe a rapid increase in temperature in plane 1 . Then, after another two minutes plane 2 is quickly heated up. Plan 3 and 4 because of a significant distance from the bale axis are heated up much longer, and the beginning of the temperature growth in these planes is observed, respectively, from 10 and 15 minutes. The maximum temperature for Plan 1 is achieved in 90 minutes of the measurement, with the continuous increase during the process of combustion, and its values was $74^{\circ} \mathrm{C}$. For subsequent planes, the temperature remains at a similar level and fluctuates around $40-50^{\circ} \mathrm{C}$, an its maximum is achieved similarly like in the case of Plane 1 , in the 90 minute of the measurement. The flue gas temperature during whole drying process varied between $80-95^{\circ} \mathrm{C}$.

\section{Numerical model}

A preliminary mathematical model was created in the ANSYS FLUENT 15.0, describing the movement of the mass and heat transfer inside the bale of straw in question. Compared to the results presented during EFM2016 the model was modified by adding evaporation-condensation model and Eulerian model. The previous model took into account only the change of the temperature field and the velocity of the drying medium flow. Currently, also the existence of 3 phases was taken into account: air, water and water vapour.

The model contains 239274 nodes and 276844 elements, mostly hexagonal. The computing grid was an average quality. This was dictated by some initial simplification for the acceleration of numerical calculations. Moreover, the transient model, SST turbulence model and Eulerian model were used. Three phases were entered in the model, which are: the air phase, the water vapour phase and the water in the liquid state phase. In addition, the bi-directional model was applied: evaporation-condensation model describing the phase transition.

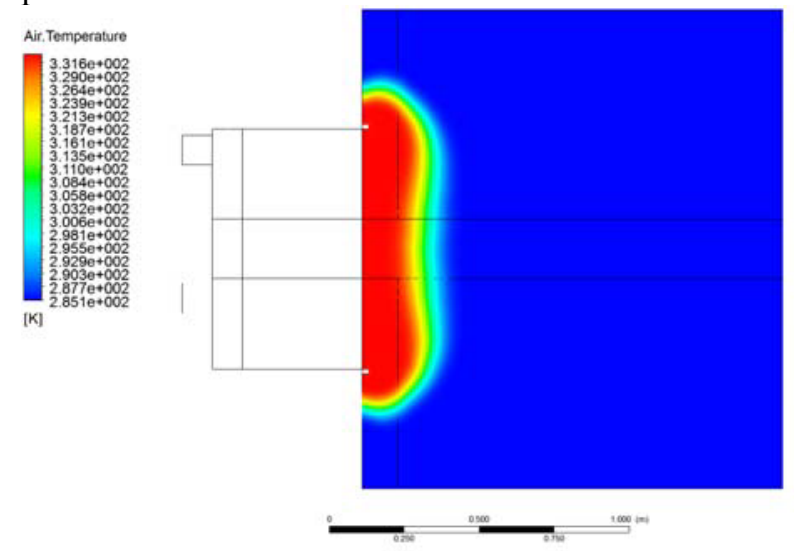

Figure 5. Temperature of the drying medium in straw bale.

The model provided two domains: 1 - intake of drying air, 2 - a bale of straw, the porous domain [18]. The time step was $1 \mathrm{~s}$, the total number of iterations 100 . The bale domain has the ability of mass and heat transfer with the surroundings. Initial conditions were: 1) temperature $-285 \mathrm{~K}, 2$ ) temperature of dry air $-333 \mathrm{~K}, 3$ ) pressure $-101325 \mathrm{~Pa}, 4)$ the volume content of water in the bale $-2 \%, 5$ ) the amount of vapour in the bale $-0 \%$. 


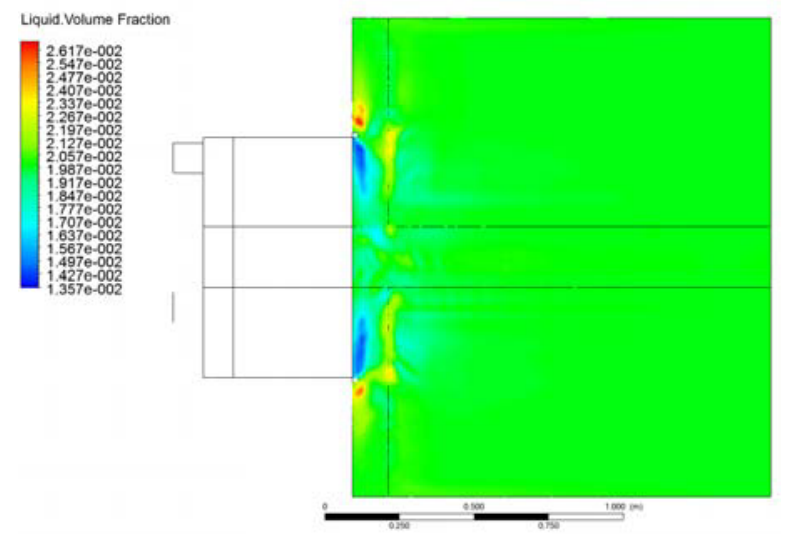

Fig. 6. Liquide volume fraction in straw bale.

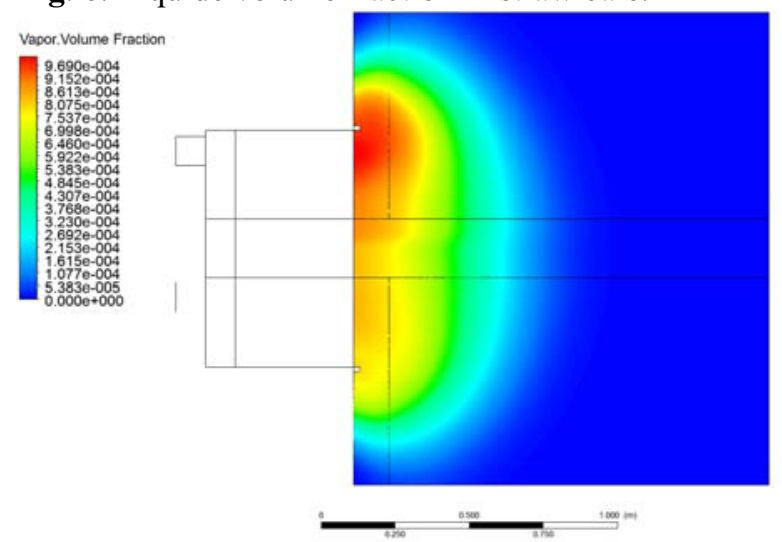

Fig. 7. Vapour volume fraction in straw bale.

Figures 5-7 present the preliminary results of the developed model. It can be observed that in the inlet portion of the drying medium there is the temperature increase of the air domain. In the area of high temperature we can observe the decrease of the water content in the bale and the increase of the water vapour content, what allows to assume that the evaporation process takes place in this place. Additionally, we can note an increased content of water in the area of high thermal gradient. In this case it can be concluded that there is the condensation of water vapour. Similar observations were made during the performance of experimental measurements. It can be assumed that this model describes the process of phase change from water into water vapour and vice versa. Currently, the model should be modified so that the injected drying medium flow through the whole model of straw, what will cause the heating of the whole bale of straw and evaporation of water from its interior. To improve the model the viscous and inertial resistance coefficients should be determined.

\section{Conclusions}

The preliminary results of the mathematical model show that the assumed objective was achieved, which was to develop the model of water evaporation and condensation of water vapour in the bale of straw. The experimental results confirm that in the place of a large temperature gradient there is a rapid increase of the moisture contents, that is, the condensation of water vapour. Currently, the works are conducted, which involve the introduction of modifications in the model, so that the process of drying takes place in the entire volume of the bale.

\section{Acknowledgment}

This work was financially supported by the statutory founding of Faculty of Energy and Fuels at AGH University of Science and Technology and it was carried out by BioORC Project (KIC InnoEnergy Programme).

\section{References}

1. E. F. Kristensen, J. K. Kristensen, Biomass Bioenerg, 26, 561 - 569 (2004)

2. J. Skvaril, A. Avelin, J. Sandberg, E. Dahlquist, Energy Procedia, 61, 643 - 647 ( 2014 )

3. L.A.C. Tarelho, E.R. Teixeira, D.F.R. Silva, R.C.E. Modolo, Energy, 90, 387-402 (2015)

4. H. Kuuluvainen, P. Karjalainen, C.J.E. Bajamundi, J. Maunula, Fuel, 139, 144-153 (2015),

5. F. J. G.Cruz, P. J. Casanova-Pelaez, J. M. PalomarCarnicero, Appl. Therm. Eng., 80, 362-373 (2015)

6. C. Gu, X. Zhang, B. Li, Z. Yuan, Powder Technol., 267, 234-239 (2014)

7. F. Geng, Z. Yuan, Y. Yan, D. Luo, H. Wang, B. Li, D. Xu, Powder Technol., 193, 50-58 (2009)

8. Yefri Chan, T. M. Nining Dyah, A. Kamaruddin, Energy Procedia, 65, 378 - 385 (2015)

9. Thermal Drying of Wet Fuels: Opportunities and Technology, Vancouver, Canada, December 1996

10. I. Ceylan, A. E. Gürel, Appl. Therm. Eng., 106, 899-905 (2016)

11. F. Geng, G. Luo, Y. Li, L. Yuan, Z. Yuan, X. Wu, Powder Technol., 267, 322-332 (2014)

12. A Review of Drying Technologies, ed. Dr Hanning Li, Dr K Finney, Sheffield, February 2010,

13. A. Alamia, H. Strom, H. Thunman, Biomass Bioenerg, 77, 92-109 (2015)

14. E.F. Zanoelo, A. Abitante, L.A.C. Meleiro, J. Food Eng., 84, 458-468 (2008),

15. F. Roman, V. Strahl-Schafer, O. Hensel, Biosyst. Eng., 112, 359-369 (2012)

16. Y. Amanlou, A. Zomorodian,, J. Food Eng., 101, 8$15(2010)$

17. F. D. Roman, O. Hensel, Biosyst. Eng., 122, 1-15 (2014)

18. W. Goryl, M. Filipowicz, EPJ Web of Conferences, 114, $02033 \quad$ (2016), DOI: http://dx.doi.org/10.1051/epjconf/201611402033

19. W Goryl, M. Filipowicz, E3S Web of Conferences (2016), In press

20. W. Goryl, Proceedings of 2nd International Conference RENEWABLE ENERGY SOURCES engineering, technology, innovations - scientific conference, Krynica, Poland (2015) 